\title{
ASPECTOS MORFOFISIOLÓGICOS EM CAMOMILA IRRIGADA DURANTE A FASE VEGETATIVA
}

\author{
Leonardo Araujo Sobral, Catariny Cabral Aleman \\ Universidade do Oeste Paulista - UNOESTE, Curso de Agronomia, Presidente Prudente - SP. E-mail: \\ catariny@unoeste.br
}

\section{RESUMO}

A Chamomilla recutita (L.) Rauschert é uma planta pertencente à família Asteraceae. É cultivada em climas temperados possuindo condições de cultivo adequadas em regiões do sul e sudeste do Brasil. Seu cultivo tem aumentado em função do amplo uso medicinal, para extração das frações de óleo essencial e flavonóides para produção de produtos farmacêuticos e cosméticos. $O$ objetivo foi avaliar a reposta fisiológica e bioquímica do desenvolvimento vegetativo das plantas de camomila. O experimento foi realizado em ambiente protegido no campus II da Unoeste, Presidente Prudente-SP. Os tratamentos aplicados foram as lâminas de irrigação: T1) 150, T2) 125, T3) 100, T4) 75, T5) 50 e T6) 25\% ETc (evapotranspiração da cultura). O delineamento experimental será inteiramente casualizado, com 6 tratamentos e 6 repetições, totalizando 36 parcelas. Foi determinado a altura de plantas, massa fresca de parte aérea e raiz, massa seca de parte aérea e raiz e concentração de prolina. Os dados foram submetidos a ANOVA e posteriormente ao teste de Tukey para comparação das médias ao nível de probabilidade de 0,05. Foi possível verificar que ocorreu maior acúmulo de massa fresca de parte aérea e raiz, e massa seca de parte aérea para a lâmina de irrigação de $75 \%$ ETc. A concentração de prolina foi maior na lâmina de irrigação de $125 \%$ ETc.

Palavras-chave: Camomila, estresse hídrico, produtividade de água, fase de desenvolvimento.

\section{MORPHOPHYSIOLOGICAL ASPECTS IN CHAMOMILE IRRIGATED DURING PHASE VEGETATIVE}

\begin{abstract}
The Chamomilla recutita (L.) Rauschert is a plant in the Asteraceae family. It is cultivated in temperate climates possessing appropriate cultivation conditions in regions of the South and Southeast of Brazil Its cultivation has increased in the light of the broad medicinal use, for the extraction of flavonoids and essential oil fractions for the production of pharmaceuticals and cosmetics. The experiment was to assess the physiological and biochemistry of Chamomile subjected to different depths of irrigation. The experiment was carried out in protected environment in campus II Unoeste, Presidente Prudente-SP were the treatments applied depths of irrigation: 150, 125, 100, 75 and 25\% ETc. (evapotranspiration of culture). The experiment was randomized design, com 6 tratements e 6 repetitions each, totaling 36 installments. The parameters was determined the height of the plants, fresh and dry matter of leafs and shoots, concentration of proline. The data will be submitted to ANOVA and later by Tukey test for comparison of medium probability of 0,05 level. The irrigation depth of $75 \%$ ETc were had the positive result in fresh and dry matter of leafs and shoot. The proline concentration was high in the irrigation depth of $125 \%$ ETc.
\end{abstract}

Keywords: Chamomile, hydric stress, water productivity, vegetative environment. 


\section{INTRODUÇÃO}

A camomila (Chamomilla recutita (L.) Rauschert) é uma planta herbácea, anual, aromática, de até um metro de altura e pertencente à família Asteraceae (LORENZI e MATOS, 2002). Esta espécie apresenta grande importância econômica para vários países. Atualmente a produção mundial desta espécie passa de 4000 toneladas ano-1 de flores secas (KAVANDI et al., 2011), produzidas principalmente nos países da Hungria, Rússia, Argentina, Alemanha, Tchecoslováquia, Finlândia, Egito e Índia (MOHAMMAD, 2011). No Brasil, a produção está situada principalmente no estado do Paraná, com produção no ano de 2011 de 929,4 toneladas de capítulos florais secos. 0 município de Mandirituba-PR é o maior produtor, e sua produtividade média corresponde a $450 \mathrm{~kg}$ ha-1 de flores secas (SEAB/DERAL, 2013). Os capítulos florais da camomila apresentam propriedades calmante, analgésica, antiespasmódica e cicatrizante, sendo empregados na indústria farmacêutica e cosmética (MAPELI et al., 2005). O suprimento artificial de água às plantas é determinado pela habilidade de utilização da água armazenada no solo e a interação com a atmosfera refere-se à combinação de fatores meteorológicos (MAROUELLI et al., 1996). O manejo adequado da água é de fundamental importância para o desenvolvimento das plantas (COSTA et al., 2002; BILIBIO et al., 2010). A água deve ser administrada nas épocas adequadas de desenvolvimento da cultura para favorecer a produção da mesma (DOORENBOS e KASSAN, 2000).

O manejo adequado da irrigação deve disponibilizar a quantidade e qualidade de água para o desenvolvimento das plantas, propiciando aumento da produtividade na colheita. É importante identificar o momento e a quantidade de água durante a irrigação para que a cultura não sofra com o excesso e nem com a falta de água nas diferentes das fases de desenvolvimento (OLIVEIRA et al., 2006). O estresse hídrico pode ser definido como todo o conteúdo de água de um tecido ou célula que está abaixo ou acima do conteúdo de água exibido no estado de hidratação adequado (TAIZ e ZEIGER, 2013).

O objetivo do presente trabalho foi avaliar aspectos morfofisiológicos e bioquímicos de plantas de camomila submetida a diferentes lâminas de irrigação, durante o desenvolvimento vegetativo.

\section{MATERIAL E MÉTODOS}

O experimento da Chamomilla recutita foi instalado na Universidade do Oeste Paulista, Faculdade de Ciências Agrárias, localizada na Rodovia Raposo Tavares km, em ambiente protegido. A instituição encontra-se a uma longitude $51^{\circ} 22^{\prime} 04^{\prime \prime}$ e latitude $22^{\circ} 07^{\prime} 04^{\prime \prime}$; aproximadamente 432 
metros do nível do mar. A região é caracterizada por um clima Subtropical úmido, com verões quentes e chuvosos e invernos secos, sendo o tipo de solo predominante Latossolo-vermelho.

As mudas de camomila (Chamomila recutita) foram preparadas utilizando sementes ISLA ${ }^{\circledR}$ em bandeja de isopor com 200 células. Após 45 DAP (dias após plantio) as mudas foram transplantadas para vasos de $11 \mathrm{~L}$ (dimensões $27 \mathrm{~cm}$ X $30 \mathrm{~cm}$ X $40 \mathrm{~cm}$ ). Os tratamentos com diferentes lâminas de irrigação foram realizados durante a fase vegetativa de desenvolvimento das plantas (45 dias após plantio até 100 dias após o plantio). 0 início da aplicação das lâminas ocorreu com pleno estabelecimento das plantas nos vasos (7 DAT - dias após transplantio). Os tratamentos consistiram em seis lâminas de irrigação determinadas pela evaporação do evaporímetro de Pichê, sendo elas 150\%, 125\%, 100\%, 75\%, 50\% e 25\% da ETc (evapotranspiração da cultura). A equação 01 e 02 foram utilizadas para determinar a lâmina de irrigação.

$$
\begin{array}{lr}
\mathrm{ETo}=\mathrm{EP} \times \mathrm{k} & \text { (equação 01) } \\
\mathrm{ETC}=\mathrm{ETo} \times \mathrm{kc} & \text { (equação 02) }
\end{array}
$$

Onde:

ETo é a evapotranspiração de referência $\left(\mathrm{mm}^{\mathrm{dia}}{ }^{-1}\right)$; EP é a evaporação de Pichê $(\mathrm{mm})$; $\mathrm{k}$ é a constante do evaporímetro (0,057); ETc é a evapotranspiração da cultura $\left(\mathrm{mm} \mathrm{dia}{ }^{-1}\right)$ e Kc é o coeficiente da cultura.

O coeficiente de cultura utilizado $(\mathrm{kc})$ foi o da hortelã de 1,0 por existirem poucos estudos referentes ao consumo hídrico de plantas medicinais.

O delineamento experimental foi inteiramente casualizados, com 6 lâminas de irrigação e 6 repetições totalizando 36 unidades experimentais. Os resultados foram submetidos a ANOVA e posteriormente ao teste Tukey ao nível de probabilidade 0,05, para comparação entre médias.

Os parâmetros avaliados foram altura das plantas, massa fresca de raiz e parte aérea, massa seca de raiz e parte aérea e a quantificação de prolina nas folhas frescas. A prolina foi determinada pela metodologia de Bates et al. (1973) modificada por Machado Neto et al. (2004).

\section{RESULTADOS E DISCUSSÃO}

De acordo com a Tabela 1, a massa fresca de parte aérea (MFPA), a massa fresca de raiz (MFR), massa seca de parte aérea (MSPA) e a concentração de prolina nas folhas frescas (PRO) apresentaram diferença estatística para as diferentes lâminas de irrigação. As alturas e a massa seca de raiz (MSR) não apresentaram diferenças para as lâminas de irrigação empregadas. 
Tabela 1. Valores de F para os parâmetros avaliados da camomila cultivada sob diferentes lâminas de irrigação.

\begin{tabular}{ccc}
\hline & Tratamentos & C.V. (\%) \\
\hline G.L. & 5 & - \\
Altura 45 DAP & $0,7143^{\text {n.s. }}$ & 15,90 \\
Altura 50 DAP & $0,9345^{\text {n.s. }}$ & 23,53 \\
Altura 55 DAP & $0,8816^{\text {n.s. }}$ & 25,04 \\
Altura 60 DAP & $1,0519^{\text {n.s. }}$ & 26,77 \\
Altura 65 DAP & $1,0261^{\text {n.s. }}$ & 21,85 \\
Altura 70 DAP & $0,9215^{\text {n.s. }}$ & 24,59 \\
Altura 75 DAP & $0,3378^{\text {n.s. }}$ & 25,94 \\
MFPA & $5,2431^{* *}$ & 23,95 \\
MFR & $3,9353^{* *}$ & 24,61 \\
MSPA & $4,3808^{* *}$ & 21,18 \\
MSR & $1,8750^{\text {n.s. }}$ & 29,10 \\
PRO & $3,5834^{*}$ & 27,08 \\
\hline
\end{tabular}

Onde: MFPA é a massa fresca de parte aérea (g); MFR é a massa fresca de raiz (g); MSPA é a massa seca de parte aérea (g); MSR é a massa seca de raiz (g) e PRO é a concentração de prolina $\left(\mu \mathrm{g} \mathrm{mg}^{-1}\right)$. As variáveis com ${ }^{*}=$ significância a $5 \%$; ${ }^{* *}=$ significância a $1 \% \mathrm{e}^{\text {n.s. }}=$ não significativos.

Na tabela 2 foi possível observar que a lâmina de irrigação de 75\% ETc apresentou o maior acúmulo de massa fresca de parte aérea. A lâmina de irrigação de 75\% ETc representa um estresse moderado. Essa lâmina atendeu as necessidades hídricas da camomila durante a fase vegetativa promovendo acumulo de biomassa fresca de parte aérea. Os dados são semelhantes ao obtidos por Pinto et al. (2014) que verificaram que para capim limão não ocorreu interferência das lâminas de irrigação no parâmetro altura, mas houve aumento da biomassa fresca em condições de déficit moderado e déficit acentuado (50\% ETc).

Tabela 2. Massa fresca de parte aérea (MFPA) das plantas de camomila submetidas a diferentes lâminas de irrigação de 150\%, 125\%, 100\%, 75\%, 50\% e 25\%ETc.

\begin{tabular}{cc}
\hline Lâmina de irrigação (ETc) & MFPA (g) \\
\hline $25 \%$ & $2,070 \mathrm{~b}$ \\
$50 \%$ & $5,476 \mathrm{~b}$ \\
$75 \%$ & $13,631 \mathrm{a}$ \\
$100 \%$ & $6,301 \mathrm{ab}$ \\
$125 \%$ & $5,080 \mathrm{~b}$ \\
$150 \%$ & $5,993 \mathrm{~b}$ \\
\hline
\end{tabular}

As letras minúsculas representam a comparação entre médias.

Na tabela 3, a lâmina de 75\% ETc apresentou maior desenvolvimento radicular, pois a mesma supriu as necessidades da planta. Isso é importante, pois, a deficiência hídrica interrompe 
o crescimento da cultura, causando uma alteração no acúmulo de massa fresca de raiz (TAIZ e ZEIGER, 2013).

Tabela 3. Massa fresca de raiz (MFR) das plantas de camomila submetidas a diferentes lâminas de irrigação de $150 \%, 125 \%, 100 \%, 75 \%, 50 \%$ e $25 \%$ ETc.

\begin{tabular}{cc}
\hline Lâmina de irrigação (ETc) & MFR (g) \\
\hline $25 \%$ & $1,420 \mathrm{~b}$ \\
$50 \%$ & $1,353 \mathrm{~b}$ \\
$75 \%$ & $3,583 \mathrm{a}$ \\
$100 \%$ & $1,683 \mathrm{ab}$ \\
$125 \%$ & $1,510 \mathrm{~b}$ \\
$150 \%$ & $2,691 \mathrm{ab}$ \\
\hline
\end{tabular}

As letras minúsculas representam a comparação entre médias.

Na tabela 4 foi observado também que a lâmina de $75 \%$ de ETc, foi a que se teve maior quantidade de massa seca da parte aérea, a água é essencial aos processos metabólicos das plantas, principalmente ao seu desenvolvimento (SANTOS e al., 2004). A deficiência hídrica pode apresentar danos severos para algumas culturas, no entanto, em algumas situações pode promover processos adaptativos que resultam no aumento do acúmulo de biomassa seca. Diferente da camomila, para o alecrim-pimenta, Alvarenga et al. (2012) constataram que a deficiência hídrica afeta diretamente o acúmulo de massa seca, provocando a redução como resposta a sensibilidade a falta de água.

Tabela 4. Massa seca de parte aérea (MSPA) das plantas de camomila submetidas a diferentes lâminas de irrigação de 150\%, 125\%, 100\%, 75\%, 50\% e 25\%ETc.

\begin{tabular}{cc}
\hline Lâmina de irrigação (ETc) & MSPA (g) \\
\hline $25 \%$ & $0,400 \mathrm{~b}$ \\
$50 \%$ & $1,365 \mathrm{ab}$ \\
$75 \%$ & $1,925 \mathrm{a}$ \\
$100 \%$ & $0,948 \mathrm{ab}$ \\
$125 \%$ & $0,650 \mathrm{~b}$ \\
$150 \%$ & $0,935 \mathrm{ab}$ \\
\hline
\end{tabular}

As letras minúsculas representam a comparação entre médias.

Foi possível observar na tabela 5 que a maior concentração de prolina. foi encontrada na lâmina de irrigação de $125 \%$ ETc. A prolina é um osmorregulador que aumenta sua concentração em resposta ao estresse hídrico. Em situações de excesso ou falta de água é possível verificar 
maior acumulo nas folhas frescas, tendo este aumento como resultado a uma adaptação ao estresse submetido.

Observa-se ainda que o excesso de água aplicado diariamente (150\% ECA) que pode causar redução imediata na troca de gases entre a planta e o ambiente podendo reduzir o suprimento de oxigênio ao sistema radicular, o que em troca pode limitar a resposta adaptativa da planta (PIZARD, 2006).

Tabela 5. Concentração de prolina nas folhas frescas das plantas de camomila submetidas a diferentes lâminas de irrigação de 150\%, 125\%, 100\%, 75\%, 50\% e 25\%ETc.

\begin{tabular}{cc}
\hline Lâmina de irrigação (ETc) & Prolina $\left.(\mu \mathrm{g} \mathrm{mg})^{-1}\right)$ \\
\hline $25 \%$ & $3,278 \mathrm{ab}$ \\
$50 \%$ & $2,078 \mathrm{~b}$ \\
$75 \%$ & $4,218 \mathrm{ab}$ \\
$100 \%$ & $2,780 \mathrm{ab}$ \\
$125 \%$ & $5,494 \mathrm{a}$ \\
$150 \%$ & $2,665 \mathrm{~b}$ \\
\hline
\end{tabular}

As letras minúsculas representam a comparação entre médias.

\section{CONCLUSÃO}

Foi possível concluir que a lâmina de irrigação de $75 \%$ da evapotranspiração da cultura (ETc) resultou no maior desenvolvimento vegetativo. Por consequência maior produção de MFPA, MSPA, MFR.

A lâmina de irrigação de $125 \%$ da evapotranspiração da cultura (ETc) proporcionou maior acúmulo de prolina.

\section{REFERÊNCIAS}

ALVARENGA, I.C.A., LOPES, O.D., PACHECO, F.V., OLIVEIRA, F.G., MARTINS, E.R. Fator resposta do alecrim-pimenta a diferentes lâminas de irrigação. Pesquisa Agropecuário Tropical, v.42, n.4, p.462-468, 2012. http://dx.doi.org/10.1590/S1983-40632012000400006

BILIBIO, C., et al. Desenvolvimento vegetativo e produtivo da berinjela submetida a diferentes tensões de água no solo. Revista Brasileira de Engenharia Agrícola e Ambiental, v.14, n.7, p.730735, 2010. http://dx.doi.org/10.1590/S1415-43662010000700007

COSTA, M. A. D., DONI FILHO, L. Aspectos do processo de produção agrícola na cultura a camomila (Chamomilla recutita (L.) Rauschert) no município de Mandirituba, Paraná. Visão Acadêmica, v.3, n.1, p.49-56, 2002.

DOORENBOS, J., KASSAN, A.H., Efeito da água no rendimento das culturas. $2^{\circ}$ ed, Campina Grande, 2000, $221 \mathrm{p}$. 
KAVANDI, H.; SHIRANI RAD, A.H.; MOUSAVI, S.A.; ESKANDARI ZANJANI K. \& BITARAFAN, Z. Plant Density Effect on Agronomic Traits of matricaria chamomilla L. under Different Nutrients Foliar Application Conditions. International Journal of Science and Advanced Technology, v.1, p.65-70, 2011.

LORENZI, H., MATOS, F.J.A. Plantas medicinais no Brasil (nativas e exóticas). São Paulo, p.147148, 2002.

MAPELI, N.C.,et al. Produção de biomassa e de óleo essencial dos capítulos florais da camomila em função de nitrogênio e fósforo. Horticultura Brasileira, v.23, n.1, 2005.

MAROUELLI, A., et al. Manejo de Irrigação em Hortaliças. Embrapa, 5 ed., Brasília,1996, 72 p. http://dx.doi.org/10.1590/S0102-05362005000100007

MOHAMMAD, S.M. Study on Cammomile (Matricaria chamomilla L.) usage and Farming. Advances in Environmental Biology, v.5, p.1446-1453, 2011.

OLIVEIRA, A.S., et al. A irrigação e a relação solo-planta-atmosfera. Brasília, 2006, 88p.

PIZARD A; SHAKIBA MR; ZEHTAB-SALMASI S; MOHAMMADI SA; DARVISHZADEH R; SAMADI A. Effect of water stress on leaf relative water content, chlorophyll, proline and soluble carbohydrates in Matricaria chamomilla L. Journal of Medicinal Plants Research, v.5, p.2483-2488, 2006.

PINTO, D.A., MANTOVANI, E.C., MELO, E.C., SEDIYAMA, G.C. VIEIRA, G.H.S. Produtividade e qualidade de óleo essencial de capim-limão, Cymbopogom citratus D.C., submetidos a diferentes lâminas de irrigação. Revista Brasileira de Plantas Medicinais, v.16, n.1, p.54-61, 2014. http://dx.doi.org/10.1590/S1516-05722014000100008

SANTOS MRA; INNECCO R. Adubação orgânica e altura de corte da erva-cidreira brasileira. Horticultura Brasileira, v.22, p.182-185, 2004. http://dx.doi.org/10.1590/S0102-05362004000200004

SEAB/DERAL. 2013.2 Disponível em: <http://www.agricultura.pr.gov.br/arquivos/File/deral/relmun1v.pdf>. Acesso 13/ago/2014.

TAIZ, L.; ZEIGER, E. Fisiologia Vegetal. 5ed., Porto Alegre, 2013, 719 p. 